VIRAL HEPATITS

\title{
Assessment of free light chains in HCV-positive patients with mixed cryoglobulinaemia vasculitis undergoing rituximab treatment
}

\author{
Umberto Basile1,*, Laura Gragnani ${ }^{2, *}$, Alessia Piluso ${ }^{2}$, Francesca Gulli $^{1}$, Teresa Urraro ${ }^{2}$, Maria T. Dell'Abate ${ }^{1}$, \\ Eleonora Torti ${ }^{1}$, Cristina Stasi ${ }^{2}$, Monica Monti ${ }^{2}$, Gian Ludovico Rapaccini ${ }^{3}$ and Anna Linda Zignego ${ }^{2}$ \\ 1 Department of Laboratory Medicine of the Catholic University of the Sacred Heart, Rome, Italy \\ 2 Center for Systemic Manifestations of Hepatitis Viruses (MaSVE), Department of Experimental and Clinical Medicine, University of Florence, \\ Florence, Italy \\ 3 Institute of Internal Medicine, Catholic University of the Sacred Heart, Rome, Italy
}

\section{Keywords}

hepatitis C virus - mixed cryoglobulinaemia -

free light chains - rituximab

\section{Abbreviation \\ CGs, cryoglobulins; CR, complete clinical response; FLC, free light chain; HCV, hepatitis C virus; IFN, interferon; IL-6, interleukin-6; LPD, lymphoproliferative disorder; MC, mixed cryoglobulinaemia; NHL, non-Hodgkin lymphoma; OR, odds ratio; PNR, partial/no response; RF, rheumatoid factor; RTX, rituximab. \\ Correspondence \\ Umberto Basile, PhD \\ Department of Laboratory Medicine - \\ Catholic University of the Sacred Heart, Largo \\ Agostino Gemelli, 8 - 00164, Rome, Italy \\ Tel: +3906 30156699 \\ Fax: +3906 30156700 \\ e-mail: umberto.basile@rm.unicatt.it}

Received 22 December 2014

Accepted 8 March 2015

Handling Editor: Stanislas Pol

DOI:10.1111/liv.12829

Liver Int. 2015; 35: 2100-2107

\begin{abstract}
Background \& Aims: Mixed cryoglobulinaemia (MC) is an HCV-related lymphoproliferative disorder characterized by the presence of circulating immune complexes called cryoglobulins. Treatment with anti-CD20 monoclonal antibody rituximab is proved to be very useful, especially in patients ineligible to interferon-based antiviral therapy. Recently, free light chain (FLC) $\kappa / \lambda$ ratio and FLC patterns were associated with MC. The aim of this study was to evaluate changes in FLC- $\kappa$, FCL- $\lambda$, FLC ratio following rituximab treatment in patients with HCV-related MC and to correlate FLC- $\kappa$, FCL- $\lambda$ and FLC ratio values with therapy response. Patients and Methods: We retrospectively enrolled 46 patients with HCV infection (26 females, 20 males), including 10 patients without signs/symptoms of MC-related vasculitis, 36 with $\mathrm{MC}$ vasculitis. Clinical and biological data were recorded at baseline and 6 months after RTX treatment. Nephelometric measurement of serum FLCs was taken. Results: The mean serum FLC- $\kappa$ level and FLC ratio were significantly higher in patients with MC, compared to HCV patients without MC and to blood donors. An abnormal FLC ratio at baseline correlated with the presence of cryoglobulins, C4 consumption, higher RF level and higher vasculitis rate. To evaluate the predictive value of FLCs, patients with MC were divided into two groups according to RTX therapy outcome (responders and no/partial responders). Abnormal baseline FLC ratio was significantly associated with no/partial response. Conclusions: RTX treatment in HCV-related MC induces a reduction in FLC- $\kappa$ and RF levels. Moreover, pretreatment FLC ratio, which can be easily assessed by a routine test, may be useful to predict response to this expensive treatment for patients with $\mathrm{HCV}$-related MC ineligible to IFN-based therapy.
\end{abstract}

Mixed cryoglobulinaemia (MC) is the most frequent, best known and strictly hepatitis $\mathrm{C}$ virus (HCV)-associated extrahepatic manifestation: in Italy, more than $90 \%$ of patients with MC are HCV positive $(1,2)$. MC is both an autoimmune and B-lymphoproliferative disorder (LPD), clinically benign but evolving to a frank malignancy in about $8-10 \%$ of cases $(2,3)$.

Mixed cryoglobulinaemia is characterized by intravascular immune complexes - the so-called cryoglobulins (CGs) - composed of IgGs and IgMs. The IgM has rheumatoid factor (RF) activity and is produced by clonally expanded autoreactive $\mathrm{B}$ cells $(4,5)$. The clinical manifes-

${ }^{\star}$ Contributed equally to this work. tations of MC are attributable to a systemic vasculitis caused by the intravascular CGs and can be classified as one of the immune complex-mediated systemic vasculitis, involving small- to medium-sized vessels. Meltzer et al. (6) firstly described MC as a triad of purpura, fatigue and arthralgia. The different symptoms arise from the involvement of various organs and systems, namely skin, joints, kidney, nervous system, salivary and lachrymal glands (6-8). Hence, the symptoms defining a fullblown MC can be so multiple and severe to determine a very poor quality of life for the patient (6-8).

Although antiviral therapy has been indicated as firstline therapy in patients with mild-to-moderate HCVrelated MC vasculitis (9), in severe cases, or in patients 


\section{Key points}

- No tools are available to predict response to RTX in patients with severe MC.

- A reliable outcome predictor is fundamental for a rational management of a more tailored therapy.

- As the latest guidelines for the new antiviral therapies indicated MC as a priority condition to treat, an additional routine test, such as the FLC dosage, useful to assess a certain diagnosis of MC, could be a new tool to identify patients who need an urgent access to antivirals.

- For patients ineligible to antivirals, FLC assay may be a predictive diagnostic tool to overcome decisions concerning RTX treatment of HCV-related MC vasculitis.

intolerant/ineligible to antiviral therapy, anti-CD20 monoclonal antibody rituximab (RTX) should be considered (10-12).

Serum free light chains (FLCs), $\kappa$ and $\lambda$, have been identified as useful markers whose alteration is linked to specific diseases; the quantitative assay of $\kappa$ chains, $\lambda$ chains and $\kappa / \lambda$ FLC ratio is a useful diagnostic tool in plasma cell dyscrasias, such as multiple myeloma, monoclonal gammopathy of undetermined significance and amyloidosis (13-19).

Furthermore, an abnormal $\kappa / \lambda$ FLC ratio is interpreted as a surrogate for clonal expansion (20-22). The fast turnover of FLC, particularly true for the $\mathrm{k}$ chain ( $<6 \mathrm{~h}$ compared to $20-25$ days to total IgG), explains that they may be considered ideal markers of response in patients undergoing RTX treatment.

Recently, an altered $\kappa / \lambda$ FLC ratio and FLC patterns were associated with the presence of MC vasculitis and/ or B-NHL in HCV-positive patients $(23,24)$. The same authors also suggested that serum FLC ratio could be used as a surrogate marker for HCV-related lymphoproliferation after IFN-based antiviral therapy (23).

To date, there are no reports in the literature concerning changes in $\kappa$ and $\lambda$ FLC and $\kappa / \lambda$ FLC ratio after administration of RTX in MC subjects.

The aim of this study was therefore to evaluate preand post-treatment $\kappa$ and $\lambda$ FLC serum values and $\kappa / \lambda$ FLC ratio levels in a cohort of patients with severe HCV-related MC vasculitis treated with RTX, due to ineligibility to other treatment strategies or since not responsive to antivirals.

\section{Patients and methods}

Patients

Forty-six patients with $\mathrm{HCV}$ infection $(26 \mathrm{~F}, 20 \mathrm{M}$, mean age $59.25 \pm 9.48$ ) were retrospectively enrolled at the MaSVE Center (Center for Systemic Manifestations of Hepatitis Viruses) outpatient clinic (University of
Florence, Florence, Italy), including 10 patients without signs/symptoms of MC-related vasculitis (pathological controls), 36 with MC vasculitis of which 3 affected by B-cell non-Hodgkin lymphoma (B-NHL). We also enrolled 50 healthy blood donors (as controls) whom had previously been tested for the absence of monoclonal components (by serum protein electrophoresis, serum and urine immunofixation).

Concerning the cohort of patients with MC, FLC assessment was performed using serum samples collected at baseline and 6 months after RTX treatment (immediately stored at $-80^{\circ} \mathrm{C}$ ). For the pathological control group, we used serum samples that were immediately stored at $-80^{\circ} \mathrm{C}$ following collection from patients with active chronic HCV infection without signs and symptoms of MC (or other autoimmune/lymphoproliferative disorders) and who had not received any antiviral treatment for at least 6 months prior blood collection.

The criteria for the diagnosis of MC vasculitis have been previously described $(10,12)$. Serum cryoglobulin levels and characterization, levels of complement fractions, rheumatoid factor and autoantibodies were evaluated as described $(25,26)$.

The diagnosis of liver disease was performed according to histological and/or clinical and ultrasound examination (27-29).

Patients with MC vasculitis enrolled in the study were not suitable for antiviral treatment, due to contraindications or poor tolerance. Inclusion criteria for RTX administration were the presence of $\mathrm{HCV}$-positive MC syndrome with advanced chronic liver disease. Exclusion criteria were all known causes of chronic liver disease other than HCV infection, with particular attention to the presence of hepatitis B surface antigen and antiHIV antibodies.

Administration of RTX was performed according to the following regimen: first intravenous infusion of $1 \mathrm{~g}$ RTX at day 0 and second perfusion of $1 \mathrm{~g}$ RTX at day 15. Prior to infusion, the patients received a premedication consisting of acetaminophen, dexchlorpheniramine maleate and allopurinol. The concomitant use of highdose steroids would be only allowed in case of hypersensitivity. Following RTX treatment, patients with MC were divided into two groups on the basis of clinical response to therapy: patients with a complete response and patients with no/partial response, according to Petrarca et al. (12).

All patients included in this study provided written informed consent in accordance with the principles of the Declaration of Helsinki and the study was approved by the University of Florence Ethical Committee.

\section{Serum FLC assessment}

Nephelometric measurement of serum FLCs in stored samples was taken by means of N Latex FLC-kappa and FLC-lambda kits (Siemens Healthcare Diagnostics Products $\mathrm{GmbH}$, Marburg, Germany), an assay that 
employs monoclonal antibodies directed against FLCs for their quantification.

As pioneer of the field, the FLC assay showed poor post-dilution linearity and relative imprecision, as well as increased probability of yielding false-negative results due to antigen excess in patients with extremely high FLC concentration. N Latex assay by Siemens, based on monoclonal antibodies and recently introduced on the European market, is demonstrated to overcome part of these problems.

Measurements were taken according to the manufacturer's protocol on the BNII automated analyzer, the lowest detection limit for $\kappa$ and $\lambda$ FLCs being $0.035 \mathrm{mg} / \mathrm{L}$. Calibrators and controls were provided by the manufacturer and consisted of stabilized human sera containing polyclonal $\kappa$ and $\lambda$ FLCs; calibrators and controls were diluted to the appropriate concentrations for serum determinations, following the manufacturer's instructions.

The analysis was performed by an operator without knowledge of the clinical information of the handled sample. Each sample was tested twice to minimize eventual discrepancies, and all tests were performed in the same laboratory with the same instrument. All safety precautions were taken while handling potentially harmful samples.

\section{Statistical analysis}

Data are expressed as mean \pm standard deviation. The numerical comparison of continuous data was made using Student's $t$-test for paired samples. Statistical significance was set at a value of $P<0.05$. The chi-square test was used to evaluate categorical variables. To assess the value of FLC-K, FCL- $\lambda$ and FLC ratio as predictors of response to therapy, the 36 patients enrolled were divided into two groups according to clinical response to treatment. Predictive values were determined by calculating odds ratio (OR).

Statistical analysis was computed using STATA 12 software (Stata Corporation, College Station, TX, USA).

\section{Results}

The main baseline characteristics of the study population with chronic HCV infection (26 F, $20 \mathrm{M}$, mean age $59.25 \pm 9.48$ years) are reported in Table 1.

FLC- $\kappa$, FCL- $\lambda$ and FLC ratio were retrospectively measured in $46 \mathrm{HCV}$ patients and in 50 blood donors.

The mean serum $\kappa$ FLC level was significantly higher in HCV-positive patients with MC vasculitis $(34.76 \pm 26.65 \mathrm{mg} / \mathrm{L})$ than in patients without MC $(17.51 \pm 9.29 \mathrm{mg} / \mathrm{L})$ and in blood donors $(13.22 \pm 5.31 \mathrm{mg} / \mathrm{L})(P=0.05$ and $P<0.0001$ respectively) (Fig. 1A).

The mean serum $\lambda$ FLC levels were alike in patients with $\mathrm{MC}$ vasculitis, without $\mathrm{MC}$ and in blood donors (Fig. 1B).
The mean FLC ratio was higher in MC vasculitis $(2.35 \pm 1.11 \mathrm{mg} / \mathrm{L})$ than in patients without $\mathrm{MC}$ $(1.072 \pm 0.22 \mathrm{mg} / \mathrm{L})$ and in blood donors $(0.81 \pm 0.24 \mathrm{mg} / \mathrm{L})(P=0.0023$ and $P=0.0008$ respectively) (Fig. 1C).

Patients were analysed according to FLC ratio values, and the whole population was virtually divided in subjects with normal ratio $(\leq 1.56)$ and with abnormal ratio $(>1.56)$. An abnormal FLC ratio correlated with the presence of CGs $(P=0.000)$ and was also associated with a higher mean cryocrit value, although statistical significance was not reached.

Patients with abnormal FLC ratio also showed a more frequent C4 consumption and higher RF levels $(P<0.005$ for both parameters), higher MC vasculitis rate $(P=0.000)$ and higher rate of Meltzer and Franklin triade (purpura $88 \%$, arthralgia $83 \%$ and asthenia $79 \%$, $P=0.004, P=0.027$ and $P=0.031$ respectively).

An involvement of the peripheral nervous system was also more frequent in the group of subjects with abnormal FLC ratio $(P=0.0021)$ as opposed to patients with an FLC ratio in the normal range.

Patients affected by vasculitis underwent RTX treatment and were divided in two subgroups according to the therapy outcome: patients with a complete clinical response (CR, $n=15)$ and patients with partial/no response (PNR, $n=21$ ).

The statistical analysis of the mean pre- and posttherapy levels of $\kappa, \lambda$ and FLC ratio among CR patients and among PNR patients did not reveal any significant difference (Table 2) as well as the comparison of the pre- and post-treatment mean values between the two response groups (Table 3). Considering the number of patients with an abnormal FLC ratio (>1.56) at baseline, this was significantly higher in the PNR compared to CR group $(P=0.0314)$.

When we considered the risk of clinical non-response to treatment, only abnormal baseline FLC ratio was significantly associated with no/partial response $(P=0.0314)$ with an OR of 4.86 (95\% CI 0.89-28.72). This result was also confirmed by multivariate analysis: indeed, abnormal FLC ratio at baseline emerged as the only independent predictive factor of no/partial response to RTX treatment (OR 7.7-95\% CI 1.17-51.06- $P=0.034$ ).

These findings are very interesting because, as previously mentioned, no tools are currently available to help clinicians predict response to RTX administration in patients with severe MC vasculitis.

\section{Discussion}

From a retrospective analysis of patients with chronic HCV infection, either with MC vasculitis or without MC, we found elevated serum levels of FLC- $\kappa$ and FLC ratio in patients with $\mathrm{MC}$ vasculitis as opposed to both the HCV-positive subjects without signs/symptoms of lymphoproliferative disorders and the 50 healthy control subjects. These results completely confirm previ- 
Table 1. Main baseline characteristic of the $46 \mathrm{HCV}$-positive patients, according to the results of FLC ratio

\begin{tabular}{|c|c|c|c|c|}
\hline & All patients $(n=46)$ & Normal FLC ratio $(n=22)$ & Abnormal FLC ratio $(n=24)$ & $P$ value \\
\hline Mean age (years) & $59.25 \pm 9.48$ & $57.16 \pm 9.22$ & $61.14 \pm 9.53$ & 0.1578 \\
\hline Sex (male/female) & $20 / 26$ & $14 / 8$ & $6 / 18$ & 0.016 \\
\hline \multicolumn{5}{|l|}{ HCV genotype } \\
\hline $1(\%)$ & 32 & 16 & 16 & 0.55 \\
\hline $2(\%)$ & 7 & 2 & 5 & 0.42 \\
\hline $3(\%)$ & 6 & 4 & 2 & 0.41 \\
\hline $4(\%)$ & 1 & - & 1 & 1.00 \\
\hline \multicolumn{5}{|l|}{ Histology } \\
\hline Chronic Hepatitis (\%) & $25(54)$ & $9(40.9)$ & $16(66.7)$ & 0.138 \\
\hline Cirrhosis (\%) & $21(46)$ & $13(59.1)$ & $8(33.3)$ & \\
\hline $\operatorname{ALT}(\mathrm{ULN})$ & $1.5 \pm 1.14$ & $2.23 \pm 1.23$ & $1.19 \pm 1.17$ & 0.0052 \\
\hline Viral titre $(\mathrm{IU} / \mathrm{ml} \times 106)$ & $3.28 \pm 3.5$ & $3.1 \pm 3.9$ & $3.4 \pm 3.2$ & 0.7761 \\
\hline \multicolumn{5}{|l|}{ Characteristics of MC } \\
\hline No MC & $10 / 46(22 \%)$ & $10 / 22(45 \%)$ & $0 / 24(0 \%)$ & 0.000 \\
\hline MCS & $36 / 46(78 \%)$ & $12 / 22(55 \%)$ & $24 / 24(100 \%)$ & 0.000 \\
\hline MCS with B-NHL & 3 & 0 & 3 & \\
\hline MCS without B-NHL & 33 & 12 & 21 & \\
\hline \multicolumn{5}{|l|}{ Characteristics of MC } \\
\hline Positive cryocrit & $36 / 46(78 \%)$ & $12 / 22(54.5 \%)$ & $24 / 24(100 \%)$ & 0.000 \\
\hline Cryocrit \% & $7.11 \pm 9.71$ & $3.21 \pm 2.35$ & $9.06 \pm 11.36$ & 0.0886 \\
\hline Type II & 23/36 (64\%) & $8 / 12(67 \%)$ & $15 / 24(62.5 \%)$ & 1.000 \\
\hline Type III & $6 / 36(17 \%)$ & $3 / 12(25 \%)$ & $3 / 24(12.5 \%)$ & 0.378 \\
\hline Type nd & $7 / 36(19 \%)$ & $1 / 12(8 \%)$ & $6 / 24(25 \%)$ & NR \\
\hline C4 consumption & $18 / 46(39 \%)$ & $5 / 22(23 \%)$ & $13 / 24(54 \%)$ & 0.0038 \\
\hline RF alteration & $30 / 46(65 \%)$ & $9 / 22(41 \%)$ & $21 / 24(88 \%)$ & 0.002 \\
\hline \multicolumn{5}{|l|}{ MC vasculitis symptoms } \\
\hline Purpura & $31 / 46(67 \%)$ & $10 / 22(45 \%)$ & $21 / 24(88 \%)$ & 0.004 \\
\hline Arthralgia & $31 / 46(67 \%)$ & $11 / 22(50 \%)$ & $20 / 24(83 \%)$ & 0.027 \\
\hline Asthenia & $29 / 46(63 \%)$ & $10 / 22(45 \%)$ & $19 / 24(79 \%)$ & 0.031 \\
\hline Sicca Syndrome & $21 / 46(46 \%)$ & $7 / 22(32 \%)$ & $14 / 24(58 \%)$ & 0.085 \\
\hline Raynaud phenomenon & $14 / 46(30 \%)$ & $4 / 22(18 \%)$ & $10 / 24(42 \%)$ & 0.114 \\
\hline Peripheral neuropathy & $33 / 46(72 \%)$ & $12 / 22(55 \%)$ & $21 / 24(88 \%)$ & 0.021 \\
\hline Renal involvement & $9 / 46(20 \%)$ & $2 / 22(9 \%)$ & $7 / 24(29 \%)$ & 0.139 \\
\hline Ulcers & $11 / 46(24 \%)$ & $4 / 22(18 \%)$ & $7 / 24(29 \%)$ & 0.497 \\
\hline \multicolumn{5}{|l|}{ Serum FLC assessment } \\
\hline$\kappa$ & $30.45 \pm 24.60$ & $17.01 \pm 13.73$ & $42.61 \pm 26.14$ & 0.002 \\
\hline$\lambda$ & $16.75 \pm 14.78$ & $17.20 \pm 17.25$ & $16.34 \pm 12.56$ & 0.8467 \\
\hline FLC ratio & $2.03 \pm 1.18$ & $1.06 \pm 0.22$ & $2.91 \pm 0.83$ & 0.0000 \\
\hline
\end{tabular}

Bold values denote significant values.

Values are expressed as mean \pm standard deviation.

ALT, alanine aminotransferase; ULN, upper limit of normal; IU, international units; nd, not determined.

FLC- $\kappa$, normal values: 6.7-22.4 mg/L; FLC- $\lambda$, normal values: 8.3-27 mg/L; FLC ratio, normal values: 0.31-1.56.

ous observations (23), suggesting a role for these serum proteins as markers of HCV-related MC vasculitis.

As expected, other classical serum parameters of $\mathrm{MC}$, namely RF and C4, were found to be altered in the $\mathrm{HCV}$ $\mathrm{MC}$ group compared to controls.

The correlations between an abnormal ratio $(>1.56)$ and presence of circulating CGs, C4 consumption, higher RF levels, higher MC vasculitis rate, higher rate of Meltzer and Franklin triade and the involvement of peripheral nervous system clearly confirm the role of this parameter as serum biomarker of disease activity.

All the patients with MC vasculitis were treated with RTX due to their ineligibility to IFN-based antiviral treatment [now recommended as the first therapeutic option for HCV-related MC in the absence of contraindications (9)] or owing to failure of previous aetiological therapies.

All the serum parameters tested at baseline were evaluated after 6 months of follow-up, when the B-cell population is considered to be restored.

Among the patients with MC treated with RTX, no statistically significant differences on the mean values of $\kappa, \lambda$ and FLC ratio were evident, between the CR and NPR groups, either at baseline or after 6 months. Notably, 6 months after RTX infusions, in the CR group, eight patients showed an abnormal ratio, while before 

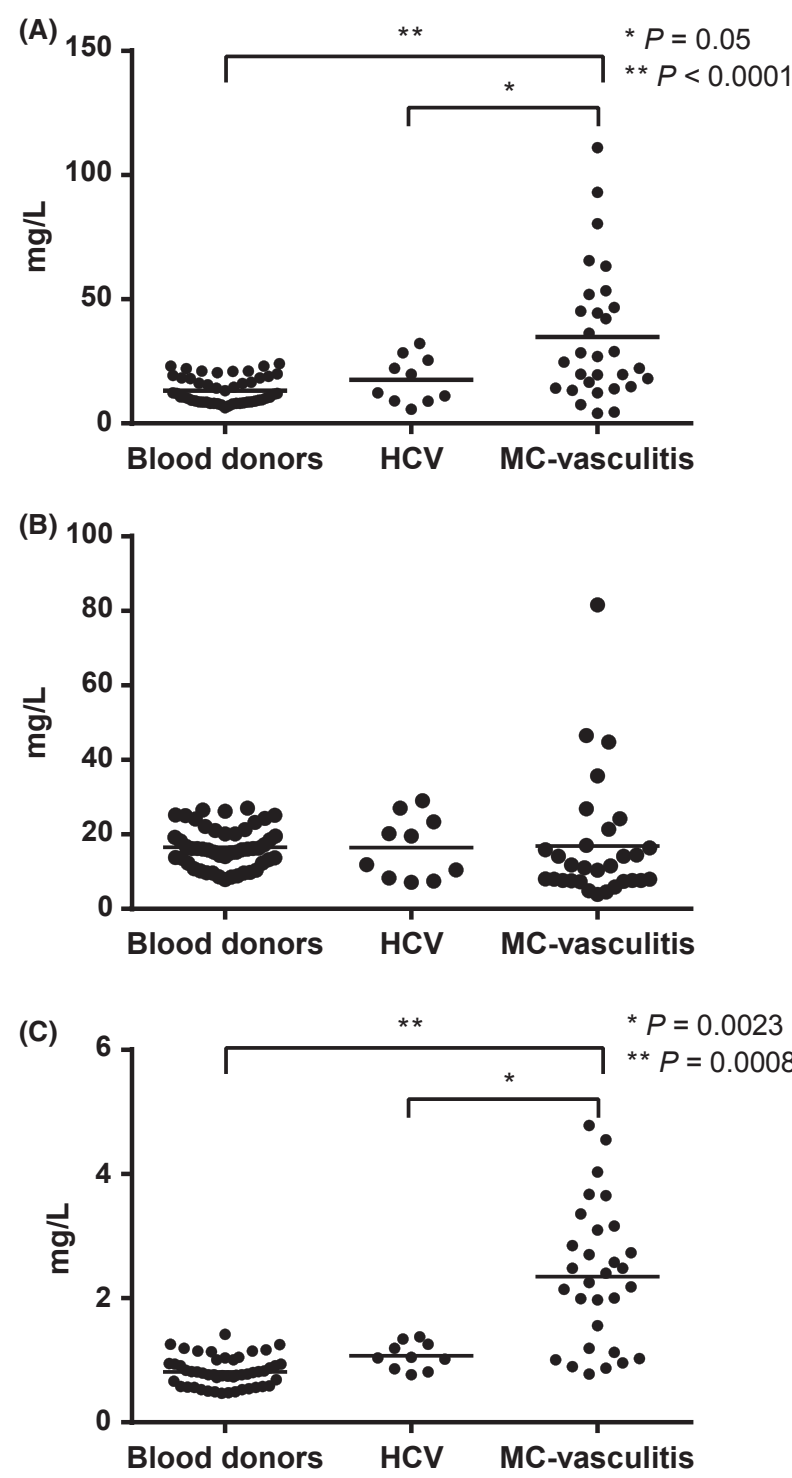

Fig. 1. Serum free light chain (FLC)-k (A), serum FLC-I levels (B) and serum FLC ratio $(C)$ in blood donors, in patients infected with hepatitis $C$ virus without any signs and/or symptoms of mixed cryoglobulinaemia (HCV) and in patients with mixed cryoglobulinaemia (MC) vasculitis.

the treatment one patient less had an abnormal ratio. The number of CR patients with abnormal $\kappa$ FLC preand post-treatment did not change.

These observations apparently seem in contrast to the role of biomarkers of disease previously attributed to $\kappa$ FLCs and FLC ratio for HCV-related MC vasculitis. Indeed, some authors reported a paradoxical increase of serum FLC concentrations in multiple myeloma treated with different drugs (30). As the effect of RTX is transitory, it is possible to hypothesize an occurring rebound effect during B-cell repopulation also in patients who achieved complete response to therapy.
In fact, a limitation of our analysis is due to its retrospective nature that restricted the study time points on the basis of biological samples available in the MaSVE Center bio-bank.

Further prospective studies will be useful to follow, in parallel, the behaviour of $\kappa$ and $\lambda$ FLC and their ratio, at different time points during the 6 months in which the B-cell compartment undergoes depletion and is subsequently followed by its slow restoration. This will allow a better understanding of the prognostic meaning of the FLCs in the free-from-disease period.

Nowadays, RTX treatment is considered effective and safe, especially in those cases of HCV-related MC vasculitis either who are ineligible for antiviral treatment or who previously failed such option. However, due to the elevated costs of RTX therapy, a correct evaluation of patients that most likely will accomplish complete response to therapy could help avoid a waste of resources, as well as spare patients from undergoing ineffective drug treatment options unsuitable for their characteristics and from side effects that, albeit rare, may still occur (i.e. hyperviscosity syndrome).

To date, a variety of predictive markers of response to RTX treatment, ranging from circulating serum proteins to genetic factors, have been proposed for different rheumatic diseases. For example, in rheumatoid arthritis, RF levels influenced the outcome of RTX treatment (31) and the anti-CCP positivity was a predictor of good to moderate response, while high Ig levels were associated with a worse outcome (32). Also, a specific host genetic background seems to have a role in determining the clinical outcome following RTX infusion. Fabris and coworkers showed that CC homozygosis of the $-174 \mathrm{G} / \mathrm{C}$ interleukin-6 (IL-6) polymorphism is linked to a lower efficacy of this treatment in patients with rheumatoid arthritis (33). The only data regarding HCV-related MC and the issue of predictors of response to RTX are reported in a study by Gragnani et al. (34) in which a particular genetic variant of Fc $\gamma$ receptor IIIA seems to be crucial to determine the anti-CD20 effectiveness.

In spite of the expectations concerning the application of these results to the clinical practice, no tools are yet available for clinicians to help them in decision-making when choosing the most appropriate individualized targeted therapy, possibly due to the fact that all the abovementioned markers still require setting-up as routine tests.

In this context, FLC nephelometric determination may be of diagnostic aid to overcome decisions concerning therapeutic treatment schemes in the light of what stated above.

Free light chains are involved in antigen recognition and binding to same extent as the intact immunoglobulins, thus contributing to the development of antibodyspecific affinity. The specific properties that link FLCs to the activation of mast cells responsible of inducing the pathogenic mechanisms of diseases characterized by chronic inflammation has raised great interest on their 
Table 2. Statistical analysis of the mean pre- and post-therapy levels of $\kappa, \lambda$ and FLC ratio among patients with complete response and among patients with partial/no response to RTX treatment

\begin{tabular}{lccc}
\hline Patients with complete response $(n=15)$ & Pretherapy & Post-therapy & $P$ value \\
\hline FLC-kappa pretherapy & $29.54 \pm 29.74$ & $29.41 \pm 29.11$ & 0.9904 \\
FLC-lambda pretherapy & $17.95 \pm 22.70$ & $17.53 \pm 24.14$ & 0.9612 \\
FLC ratio pretherapy & $2.02 \pm 1.01$ & $2.23 \pm 1.02$ & 0.5755 \\
\hline Patients with partial/no response $(n=21)$ & Pretherapy & Post-therapy & $P$ value \\
\hline FLC-kappa post-therapy & $38.24 \pm 24.64$ & $39.49 \pm 27.45$ & 0.8774 \\
FLC-lambda post-therapy & $16.13 \pm 11.35$ & $15.61 \pm 14.20$ & 0.8964 \\
FLC ratio post-therapy & $2.57 \pm 1.15$ & $3.26 \pm 2.29$ & 0.2244 \\
\hline
\end{tabular}

Table 3. Comparison of the pre- and post-treatment mean values between patients with complete response and patients with partial/no response

\begin{tabular}{lccc}
\hline & $\begin{array}{l}\text { Patients with } \\
\text { complete response } \\
(n=15)\end{array}$ & $\begin{array}{l}\text { Patients with } \\
\text { partial/no response } \\
(n=21)\end{array}$ & $P$ value \\
\hline FLC-kappa pretreatment & $29.54 \pm 29.74$ & $38.24 \pm 24.64$ & 0.3447 \\
FLC-lambda pretreatment & $17.95 \pm 22.70$ & $16.13 \pm 11.35$ & 0.7530 \\
FLC ratio pretreatment & $2.02 \pm 1.01$ & $2.57 \pm 1.15$ & 0.1464 \\
FLC-kappa post-treatment & $29.41 \pm 29.11$ & $39.49 \pm 27.45$ & 0.2969 \\
FLC-lambda post-treatment & $17.53 \pm 24.14$ & $15.61 \pm 14.20$ & 0.7661 \\
FLC ratio post-treatment & $2.23 \pm 1.02$ & $3.26 \pm 2.29$ & 0.1133 \\
\hline
\end{tabular}

clinical use, both as challenging 'target' and as therapeutic marker of biochemical progression or remission of the disease. Indeed, the clinical interest for the determination of FLC in patients infected with human immunodeficiency virus (HIV) or hepatitis $\mathrm{C}$ virus (HCV) was reached based on the observations that an increased concentration of FLCs in these diseases may be considered as biomarker for early onset of neoplasia, in particular of lymphoma (35).

The development of serum assays for free FLC- $\kappa$ and FLC- $\lambda$ has opened the door to new applications increasing their clinical importance beyond monoclonal gammopathies and plasma cell dyscrasias. In particular, the availability of these tests may open new perspectives for diagnosing and managing various diseases characterized by chronic inflammation (36).

For most of these diseases, serum polyclonal FLC concentrations correlate with disease activity, therefore constituting a potentially valuable tool for adjusting therapeutic regimens.

Furthermore, the FLC assay is a routine test which enables easy performance before RTX infusion to select patients with greater chances of success. At the same time, subjects who stand less chances of being complete responders could be addressed towards other therapies. The availability of reliable outcome predictors is fundamental for a more rational management of economic resources and for a more tailored therapy.

On the other hand, the importance of the extrahepatic manifestations of $\mathrm{HCV}$ is nowadays officially rec- ognized and the latest AASLD guidelines for the new direct acting antivirals (DAA) indicated the MC among the highest priority conditions to treat because of the risk for severe complications (37). Therefore, the availability of an additional routine test, such as the FLC dosage, useful to assess a diagnosis of MC, could be a new tool to identify patients who need an urgent access to antiviral treatment. Also, in the era of the new highly effective DAA, the availability of this non-invasive test could be precious to assess the effective control of HCV-related lymphoproliferations after viral eradication.

Furthermore, the low cost of the FLC assay (the price is comparable to the other routine tests currently used as autoimmunity markers) represents an additional positive feature.

Further prospective studies conducted on wider cohorts of patients performed by monitoring serum FLC levels at different time points after RTX administration will help to set up a new prognostic test useful for predicting therapy outcome in patients affected by HCV-related MC vasculitis and to understand how FLC levels follow the timing of B-cell repopulation.

\section{Acknowledgements}

Financial support: L. G. is supported by a 2015 fellowship 'Fondazione Umberto Veronesi'; A. P. is supported by a F.I.R.E. fellowship. 
Conflict of interest: The authors do not have any disclosures to reports.

\section{References}

1. Misiani R, Bellavita P, Fenili D, et al. Hepatitis C virus infection in patients with essential mixed cryoglobulinemia. Ann Intern Med 1992; 117: 573-7.

2. Zignego AL, Gragnani L, Giannini C, Laffi G. The hepatitis $\mathrm{C}$ virus infection as a systemic disease. Intern Emerg Med 2012; 7(Suppl 3): S201-8.

3. Vallat L, Benhamou Y, Gutierrez M, et al. Clonal B cell populations in the blood and liver of patients with chronic hepatitis C virus infection. Arthritis Rheum 2004; 50: 3668-78.

4. Sansonno D, De Vita S, Iacobelli AR, et al. Clonal analysis of intrahepatic B cells from $\mathrm{HCV}$-infected patients with and without mixed cryoglobulinemia. J Immunol 1998; 160: 3594-601.

5. Knight GB, Gao L, Gragnani L, et al. Detection of WA B cells in hepatitis $\mathrm{C}$ virus infection: a potential prognostic marker for cryoglobulinemic vasculitis and B cell malignancies. Arthritis Rheum 2010; 62: 2152-9.

6. Meltzer M, Franklin EC, Elias K, McCluskey RT, Cooper N. Cryoglobulinemia-a clinical and laboratory study. II. Cryoglobulins with rheumatoid factor activity. Am J Med 1966; 40: 837-56.

7. Saccardo E, Novati P, Sironi D, et al. Causes of death in symptomatic cryoglobulinemia: 30 years of observation in a Department of Internal Medicine. Dig Liver Dis 2007; 39 (Suppl 1): S52-4.

8. Ramos-Casals M, Stone JH, Cid MC, Bosch X. The cryoglobulinaemias. Lancet 2012; 379: 348-60.

9. Pietrogrande M, De Vita S, Zignego AL, et al. Recommendations for the management of mixed cryoglobulinemia syndrome in hepatitis C virus-infected patients. Autoimmun Rev 2011; 10: 444-54.

10. De Vita S, Quartuccio L, Isola M, et al. A randomized controlled trial of rituximab for the treatment of severe cryoglobulinemic vasculitis. Arthritis Rheum 2012; 64: 843-53.

11. Zaja F, De Vita S, Mazzaro C, et al. Efficacy and safety of rituximab in type II mixed cryoglobulinemia. Blood 2003; 101: 3827-34.

12. Petrarca A, Rigacci L, Caini P, et al. Safety and efficacy of rituximab in patients with hepatitis $C$ virus-related mixed cryoglobulinemia and severe liver disease. Blood 2010; 116: 335-42.

13. Anderson KC, Alsina M, Bensinger W, et al. Multiple myeloma. J Natl Compr Canc Netw 2011; 9: 1146-83.

14. Bird JMOR, D'Sa S, Snowden JA, et al. Haemato-oncology Task Force of the British Committee for Standards in Haematology (BCSH), UK Myeloma Forum. Guidelines for the Diagnosis and Management of Multiple Myeloma 2013. London, UK: British Committee for Standards in Haematology (BCSH), 2013.

15. Bird J, Behrens J, Westin J, et al. UK Myeloma Forum (UKMF) and Nordic Myeloma Study Group (NMSG): guidelines for the investigation of newly detected M-proteins and the management of monoclonal gammopathy of undetermined significance (MGUS). Br J Haematol 2009; 147: $22-42$.
16. Guidelines on the diagnosis and management of AL amyloidosis. Br J Haematol 2004; 125: 681-700.

17. Bird JM, Owen RG, D'Sa S, et al. Guidelines for the diagnosis and management of multiple myeloma 2011. Br J Haematol 2011; 154: 32-75.

18. Dimopoulos M, Kyle R, Fermand JP, et al. Consensus recommendations for standard investigative workup: report of the International Myeloma Workshop Consensus Panel 3. Blood 2011; 117: 4701-5.

19. Dispenzieri A, Kyle R, Merlini G, et al. International Myeloma Working Group guidelines for serum-free light chain analysis in multiple myeloma and related disorders. Leukemia 2009; 23: 215-24.

20. Katzmann JA, Clark RJ, Abraham RS, et al. Serum reference intervals and diagnostic ranges for free kappa and free lambda immunoglobulin light chains: relative sensitivity for detection of monoclonal light chains. Clin Chem 2002; 48: $1437-44$.

21. Bradwell AR, Carr-Smith HD, Mead GP, Harvey TC, Drayson MT. Serum test for assessment of patients with Bence Jones myeloma. Lancet 2003; 361: 489-91.

22. Rajkumar SV, Kyle RA, Therneau TM, et al. Serum free light chain ratio is an independent risk factor for progression in monoclonal gammopathy of undetermined significance. Blood 2005; 106: 812-7.

23. Terrier B, Sene D, Saadoun D, et al. Serum-free light chain assessment in hepatitis C virus-related lymphoproliferative disorders. Ann Rheum Dis 2009; 68: 89-93.

24. Geri G, Terrier B, Semoun O, et al. Surrogate markers of $B$ cell non-Hodgkin's lymphoma in patients with hepatitis C virus-related cryoglobulinaemia vasculitis. Ann Rheum Dis 2010; 69: 2177-80.

25. Zignego AL, Giannelli F, Marrocchi ME, et al. $\mathrm{T}(14 ; 18)$ translocation in chronic hepatitis $\mathrm{C}$ virus infection. Нераtology 2000; 31: 474-9.

26. Andreone P, Zignego AL, Cursaro C, et al. Prevalence of monoclonal gammopathies in patients with hepatitis $\mathrm{C}$ virus infection. Ann Intern Med 1998; 129: 294-8.

27. Bedossa P, Poynard T. An algorithm for the grading of activity in chronic hepatitis C. The METAVIR Cooperative Study Group. Hepatology 1996; 24: 289-93.

28. Gaiani S, Gramantieri L, Venturoli N, et al. What is the criterion for differentiating chronic hepatitis from compensated cirrhosis? A prospective study comparing ultrasonography and percutaneous liver biopsy. J Hepatol 1997; 27: 979-85.

29. Stasi C, Arena U, Vizzutti F, et al. Transient elastography for the assessment of liver fibrosis in patients with chronic viral hepatitis: the missing tool? Dig Liver Dis 2009; 41: 863-6.

30. Dawson MA, Patil S, Spencer A. Extramedullary relapse of multiple myeloma associated with a shift in secretion from intact immunoglobulin to light chains. Haematologica 2007; 92: 143-4.

31. Maneiro RJ, Salgado E, Carmona L, Gomez-Reino JJ. Rheumatoid factor as predictor of response to abatacept, rituximab and tocilizumab in rheumatoid arthritis: systematic review and meta-analysis. Semin Arthritis Rheum 2013; 43: 9-17.

32. Couderc M, Mathieu S, Pereira B, Glace B, Soubrier M. Predictive factors of rituximab response in rheumatoid arthritis: results from a French university hospital. Arthritis Care Res (Hoboken) 2013; 65: 648-52. 
33. Fabris M, Quartuccio L, Lombardi S, et al. The CC homozygosis of the $-174 \mathrm{G}>\mathrm{C}$ IL-6 polymorphism predicts a lower efficacy of rituximab therapy in rheumatoid arthritis. Autoimmun Rev 2012; 11: 315-20.

34. Gragnani L, Piluso A, Giannini C, et al. Genetic determinants in hepatitis $C$ virus-associated mixed cryoglobulinemia: role of polymorphic variants of BAFF promoter and Fcgamma receptors. Arthritis Rheum 2011; 63: 1446-51.
35. Bibas M, Lorenzini P, Cozzi-Lepri A, et al. Polyclonal serum-free light chains elevation in HIV-infected patients. AIDS 2012; 26: 2107-10.

36. Basile U, Mussap M. Rationale and prospects of serum immunoglobulin free light chain (FLC) determination in chronic inflammatory disease. Biochimica Clinica 2013; 37: 357-64.

37. AASLD/IDSA/IAS-USA. Recommendations for testing, managing, and treating hepatitis C. 2014; Retrieved April 24, 2014. 